\title{
Paradoxa und Antinomien
}

\section{Hans Stalder}

Prof. Dr. med., Redaktionsmitglied

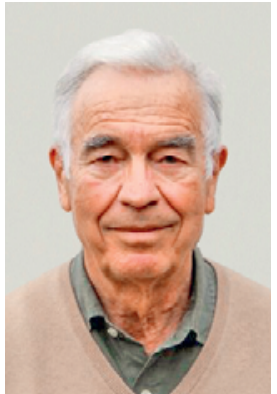

Sie haben es gewiss gelesen: Google und Co. investieren in den Vereinigten Staaten Millionen im Bereich des Transhumanismus. Sie hoffen unter anderem, die Lebenserwartung auf 200 Jahre, ja sogar 1000 Jahre zu steigern, und träumen sogar von der Unsterblichkeit. Ist es nicht paradox, dass gleichzeitig in den letzten Jahren die Lebenserwartung in den Vereinigten Staaten gesunken ist? Sie ist niedriger als auf Kuba. Bei uns haben wir in diesem Bereich ein anderes Paradoxon, gewissermassen umgekehrt: In der Schweiz werden die Menschen immer älter, aber die Nachfrage nach Suizidhilfe steigt kontinuierlich, und bei EXIT wächst das Vermögen.

Beim Nachdenken finden sich in der Medizin und in unserem Gesundheitssystem problemlos ein Dutzend Paradoxa und Widersprüchlichkeiten, und ich bin sicher, dass Ihnen noch weitere einfallen werden. Hier einige Beispiele:

Die elektronische Datenverarbeitung hätte die Arbeit der Assistentinnen und Assistenten erleichtern sollen. Tatsache ist jedoch, dass diese jetzt mehr als die Hälfte der Zeit am Computer und immer weniger Zeit mit ihren Patientinnen und Patienten verbringen.

Mit dem Argument der Kostenreduzierung möchte die Mehrheit im Parlament die Franchisen erhöhen: In der Schweiz ist jedoch der Selbstbehalt bereits jetzt so hoch wie nirgendwo sonst, und vieles weist heute darauf hin, dass dieser die Gesundheitskosten gesamthaft erhöht.

Die Kantone unterstützen gut ein Drittel der Bevölkerung bei der Zahlung ihrer Beiträge für die obligatorische Krankenpflegeversicherung. Dies bringt beträchtliche Verwaltungskosten mit sich. Und doch bleibt die Schweiz das einzige Land, das nicht die naheliegende Lösung für dieses Problem gewählt hat: die Anpassung der Prämien an das Einkommen.

Die DRG hätten die Behandlungskosten reduzieren sollen. Resultat: Sie haben die Verwaltungskosten deutlich erhöht.

Seit etwa 20 Jahren steigen die Krankenkassen-Beiträge durchschnittlich um 3 Prozent pro Jahr. Dies wird häufig damit begründet, dass die Menschen immer älter werden. Nun steigt die Lebenserwartung aber nicht jedes Jahr um 3 Prozent, denn sonst läge sie aktuell bei 125 Jahren. Wir werden also immer älter, aber die Normalwerte in der Medizin (Cholesterin, Blutdruck, Knochendichte usw.) entsprechen immer noch denen von Zwanzigjährigen. Somit findet man immer seltener ältere Personen, die der Norm entsprechen.

In einem Interview (NZZ, 18.2.2019) äussern der Rektor der Universität Zürich und der Vizepräsident der ETH sich begeistert über den neuen Bachelor-Studiengang in Medizintechnik der ETH, ihr Gespräch endet jedoch mit einer Lobrede auf empathische Allgemeinmediziner. Die Preise für Medikamente in der Schweiz sind im weltweiten Vergleich sehr hoch. Es genügt, über die Grenze zu fahren, um sie günstiger zu erhalten, dann aber werden sie von den Kassen nicht erstattet.

Bezüglich der Medikamente: Es kommen neue Wirkstoffe auf den Markt, die individuell auf einen einzigen Patienten zugeschnitten sind und mehrere Hunderttausend Franken kosten. Gleichzeitig mangelt es an günstigen Impfstoffen und Antibiotika für die Allgemeinheit. Unser Gesundheitssystem wird oft als eines der besten, wenn nicht sogar das beste betrachtet. Und doch verzichtet ein Fünftel der Schweizer Bevölkerung aus finanziellen Gründen auf medizinische Versorgung.

Die schädlichen Auswirkungen des Rauchens sind wohlbekannt, aber die Schweiz ist nicht in der Lage, die Empfehlungen der WHO umzusetzen.

Früher haben wir mehr als 60 Stunden pro Woche gearbeitet, ohne den Nachtdienst mitzurechnen, aber niemand sprach von Burnout - es gab nicht einmal den Begriff.

Man hat Mühe, wissenschaftlich nachzuweisen, dass Alternativmedizin bessere Ergebnisse erzielt als Placebos. Und doch setzen hauptsächlich Personen mit einer Hochschulausbildung auf alternative Angebote.

Das wären einige Beispiele für Paradoxa. Natürlich werden Sie für jedes eine oder mehrere Erklärungen finden. Übrigens: Auch in anderen Bereichen gibt es viele Widersprüchlichkeiten. Ist es zum Beispiel nicht paradox, dass unsere Städte und unser ganzes Land einerseits Musterschüler im Hinblick auf nachhaltige Energien sein möchten, andererseits aber Kampagnen durchführen, um Touristen aus Asien anzuziehen? Denkt man, dass sie mit dem Fahrrad oder zu Fuss zu uns kommen? Oder dass die Skilifte, Helikopterrundflüge oder Schneekanonen keine Energie verbrauchen? 